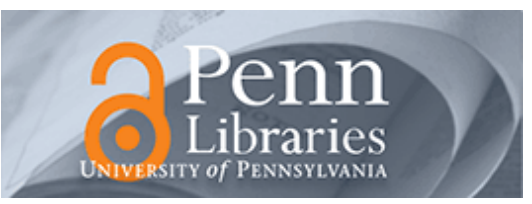

University of Pennsylvania

ScholarlyCommons

September 2004

\title{
Level Sets and Stable Manifold Approximations for Perceptually Driven Nonholonomically Constrained Navigation
}

\author{
Gabriel A. D. Lopes \\ University of Michigan \\ Daniel E. Koditschek \\ University of Pennsylvania, kod@seas.upenn.edu
}

Follow this and additional works at: https://repository.upenn.edu/ese_papers

\section{Recommended Citation \\ Gabriel A. D. Lopes and Daniel E. Koditschek, "Level Sets and Stable Manifold Approximations for Perceptually Driven Nonholonomically Constrained Navigation", . September 2004.}

Copyright 2004 IEEE. Reprinted from Proceedings of the 2004 IEEE/RSJ International Conference on Intelligent Robots and Systems (IROS 2004), Volume 2, pages 1481-1486.

This material is posted here with permission of the IEEE. Such permission of the IEEE does not in any way imply IEEE endorsement of any of the University of Pennsylvania's products or services. Internal or personal use of this material is permitted. However, permission to reprint/republish this material for advertising or promotional purposes or for creating new collective works for resale or redistribution must be obtained from the IEEE by writing to pubs-permissions@ieee.org. By choosing to view this document, you agree to all provisions of the copyright laws protecting it.

NOTE: At the time of publication, author Daniel Koditschek was affiliated with the University of Michigan. Currently (August 2005), he is a faculty member in the Department of Electrical and Systems Engineering at the University of Pennsylvania.

This paper is posted at ScholarlyCommons. https://repository.upenn.edu/ese_papers/131

For more information, please contact repository@pobox.upenn.edu. 


\title{
Level Sets and Stable Manifold Approximations for Perceptually Driven Nonholonomically Constrained Navigation
}

\author{
Abstract \\ This paper addresses problems of robot navigation with nonholonomic motion constraints and \\ perceptual cues arising from onboard visual servoing in partially engineered environments. We focus on a \\ unicycle motion model and a variety of artificial beacon constellations motivated by relevance to the \\ autonomous hexapod, RHex. We propose a general hybrid procedure that adapts to the constrained \\ motion setting the standard feedback controller arising from a navigation function in the fully actuated \\ case by switching back and forth between moving "down" and "across" the associated gradient field \\ toward the stable manifold it induces in the constrained dynamics. Guaranteed to avoid obstacles in all \\ cases, we provide some reasonably general sufficient conditions under which the new procedure \\ guarantees convergence to the goal. Simulations are provided for perceptual models previously \\ introduced by other authors.

\section{Comments} \\ Copyright 2004 IEEE. Reprinted from Proceedings of the 2004 IEEE/RSJ International Conference on \\ Intelligent Robots and Systems (IROS 2004), Volume 2, pages 1481-1486. \\ This material is posted here with permission of the IEEE. Such permission of the IEEE does not in any way \\ imply IEEE endorsement of any of the University of Pennsylvania's products or services. Internal or \\ personal use of this material is permitted. However, permission to reprint/republish this material for \\ advertising or promotional purposes or for creating new collective works for resale or redistribution must \\ be obtained from the IEEE by writing to pubs-permissions@ieee.org. By choosing to view this document, \\ you agree to all provisions of the copyright laws protecting it. \\ NOTE: At the time of publication, author Daniel Koditschek was affiliated with the University of Michigan. \\ Currently (August 2005), he is a faculty member in the Department of Electrical and Systems Engineering \\ at the University of Pennsylvania.
}




\section{Level Sets and Stable Manifold Approximations for Perceptually Driven Nonholonomically Constrained Navigation}

\author{
Gabriel A. D. Lopes \\ EECS Department, College of Engineering \\ University of Michigan, Ann Arbor, MI \\ Email: glopes@umich.edu
}

\author{
Daniel E. Koditschek \\ EECS Department, College of Engineering \\ University of Michigan, Ann Arbor, MI \\ Email: kod@umich.edu
}

\begin{abstract}
This paper addresses problems of robot navigation with nonholonomic motion constraints and perceptual cues arising from onboard visual servoing in partially engineered environments. We focus on a unicycle motion model and a variety of artificial beacon constellations motivated by relevance to the autonomous hexapod, RHex. We propose a general hybrid procedure that adapts to the constrained motion setting the standard feedback controller arising from a navigation function in the fully actuated case by switching back and forth between moving "down" and "across" the associated gradient field toward the stable manifold it induces in the constrained dynamics. Guaranteed to avoid obstacles in all cases, we provide some reasonably general sufficient conditions under which the new procedure guarantees convergence to the goal. Simulations are provided for perceptual models previously introduced by other authors.
\end{abstract}

\section{INTRODUCTION}

The literature on navigation of nonholonomically constrained bodies is extensive. Most work has been focused on systems with no sensory constraints. Khennouf et al. [9] and Luo et al. [13] use invariant manifolds; Astlofi [1], makes the system discontinuous and stabilizes it by continuous feedback control; Tayebi et al. [21] use back stepping design; Monaco et al. [15] apply multi-rate digital control; Sordalen [20], Pomet [17] and Samson [18] propose time varying feedback control laws.

In general, applying a smooth feedback control law to a nonholonomically constrained system introduces a center manifold in the configuration space. The goal lies on the center manifold and attracts all initial conditions on its (generically transverse) co-dimension one stable manifold (a leaf of the foliation [6] generated by the constraints). Ikeda et al, [7] introduced the notion of Variable Constraint Control (VCC) in which a feedback controller is designed to achieve an invariant manifold that goes through the goal, in effect, picking out a distinguished trajectory lying within the goal's stable manifold. The elegant formulation allows reaching the goal in two steps but has some shortcomings: the first step aims only at a specific, one-dimensional trajectory, instead of the entire goal's co-dimension one stable manifold. Moreover, it is not obvious how to integrate perceptual limitations in the resulting control law.

Other authors focus specifically on problems of perceptual limitation. Ostrowsky [22] uses a blimp equipped with a camera, maintaining a ball on the center of the camera's field of view. Chaumette [14] positions a fully actuated camera arm in relation to a collection of features. Cowan [4] uses navigation functions [10] to position a 6dof arm.

More recently Murrieri et al. [16] and Kantor et al. [8] combine both motion constraints with perceptual limitation. Both authors assume a particular set of nonholonomic constraints. Then, a feedback control law is built taking into account those constraints. Kantor et al. extend Ikeda's work by using a sequential composition of controllers to reach a zone where it is safe to apply VCC. This approach can result in optimized trajectories but can be hard to reuse on systems with different motion models and/or different perceptual constraints.

In this paper, we seek to decouple the (typically holonomic) perceptual constraints from the (typically nonholonomic) motor constraints by adapting an "arbitrary" navigation function [10] to an "arbitrary" nonholonomically constrained first order mechanism operating in the configuration space comprising the navigation function's domain. The encoding of holonomic constraints via navigation functions is a very effective means of constructing "designer" basins around specified goal points for fully actuated first and second order mechanisms. For example, in visual servoing applications, the navigation function takes into account external constraints like limited field of view, obstacles and so on. We are particularly interested in extending Cowans work on navigation with visual beacons to the robot RHex [19], but we will introduce a considerably more general framework for solving such problems.

We introduce a two step controller: the first moves on level sets of the gradient function so as to reach the goal's stable manifold; the second uses the gradient control law to reach the goal. If, as is generally the case, a closed form representation of the stable manifold cannot be found, an approximation can be used. In any case, by iterating successive applications of both controllers the robot is guaranteed, under fairly general conditions, to reach the goal without hitting any obstacle along the way. 


\section{Control Law}

A. Adapting navigation functions to noholonomically constrained systems

As in [7] consider the first order drift free underactuated system described by:

$$
\dot{q}=B(q) u ; \quad q \in \mathcal{D} \subset \mathbb{R}^{n}, u \in \mathbb{R}^{m},
$$

Where $u \in \mathbb{R}^{m}$ are the velocity inputs of the system and $\mathcal{D}$ is a compact set. Suppose that $B: \mathcal{D} \rightarrow \mathbb{R}^{n} \times \mathbb{R}^{m}$ is rank $m<n$. Define the nonholonomic projection matrix $M: \mathcal{D} \rightarrow \mathbb{R}^{n}$ as follows, where $B(q)^{\dagger}=\left(B^{T} B\right)^{-1} B^{T}$ is the pseudo-inverse of $B(q)$ :

$$
M(q)=B(q) B(q)^{\dagger}=B(q)\left(B(q)^{T} B(q)\right)^{-1} B(q)^{T}
$$

$M$ can be interpreted as a projection into the available directions of motion with constraints defined by $B$. Then if we let $u=B(q)^{\dagger} v$ rewrite equation (1) as:

$$
\dot{q}=M(q) v ; \quad v \in \mathbb{R}^{n}
$$

Suppose the control Lie algebra [2] on $M$ spans $\mathbb{R}^{n}$ on the configuration space. Now consider the navigation function $\varphi: \mathcal{D} \rightarrow \mathbb{R}$ such that the following system is globally asymptotically stable at the goal $q^{*}$ :

$$
\dot{q}=-\nabla \varphi(q)
$$

A Navigation function [10] is a $C^{2}$ artificial potential function on a compact manifold $\mathcal{D}$, such that $\varphi: \mathcal{D} \rightarrow$ $[0,1]$. It must encode a goal set $\mathcal{G}$ as the unique global minimum, $\varphi(\mathcal{G})=0$, and achieve a maximum of 1 on the entire boundary of $\mathcal{D}$, i.e., $\varphi(\partial \mathcal{D})=1$. (For more information on how to construct Navigation functions see [10]). Apply $\varphi$ to equation (3) to get:

$$
\dot{q}=-M(q) \nabla \varphi(q)
$$

Define the set $\mathcal{W}^{c}=\{q \in \mathcal{D}: M(q) \nabla \varphi(q)=0\}$. Using $\varphi$ as a Lyapunov function on system (5), and noticing that $M$ by construction is a positive semi-definite matrix, the derivative of the Lyapunov function is negative outside $\mathcal{W}^{c}$ and zero at $\mathcal{W}^{c}$

$$
\frac{d \varphi}{d t}=-\nabla \varphi(q)^{T} M(q) \nabla \varphi(q)\left\{\begin{array}{cc}
<0, & \forall q \notin \mathcal{W}^{c} \\
=0, & \forall q \in \mathcal{W}^{c}
\end{array}\right.
$$

By La Salle's theorem every solution of (5) approaches $\mathcal{Q} \subset \mathcal{W}^{c}$, where $\mathcal{Q}$ is the largest invariant set of $\mathcal{W}^{c}$ at some fixed level, $\Phi_{\alpha}:=\varphi^{-1}[\alpha]$, of $\varphi$. In this case $\mathcal{Q}=\mathcal{W}^{c}$ by definition of $\mathcal{W}^{c}$. Moreover, the Center Manifold Theorem for Flows [6] shows that if $\varphi$ is $C^{r}$ then system (5) has a $C^{r}$ invariant stable manifold $\mathcal{W}^{s}$ at $q^{*}$ of dimension $m$ and a $C^{r-1}$ center manifold of dimention $n-m$, in this case $\mathcal{W}^{c}$.

\section{B. Moving on a level set of $\varphi$}

Since (3) drives the robot to a point on $\mathcal{W}^{c}$ that will generally be removed from the goal, we find a controller that first reaches the stable manifold $\mathcal{W}^{s}$ by moving on level sets $\Phi_{\alpha}$. By doing so the robot is guaranteed not to hit the obstacles.
Suppose we can find a vector field $f$ such that:

$$
f: \mathcal{D} \rightarrow T \mathcal{D} \quad \mid \quad f(q) \in T L^{\prime}(q) \cap \Im(M)
$$

Where $T L^{\prime}(q)$ is the tangent space of the level sets of $\varphi$ at $q$ and $\Im(M)$ is the image of $M$. By construction, $\varphi(q)=$ const is an invariant sub-manifold of the system $\dot{q}=f(q)$. To see this, we simply take the total derivative of $\varphi$ and note that $\dot{\varphi}=\nabla \varphi \cdot \dot{q}=\nabla \varphi \cdot f$ and $f \in T L^{\prime}(q) \cap \Im(M) \Rightarrow$ $f \in T L^{\prime}(q)$. Hence, by definition of the orthogonal complement $\nabla \varphi \in\left(T L^{\prime}(q)\right)^{\perp} \Rightarrow \nabla \varphi \cdot f=0 \Rightarrow \dot{\varphi}=0$.

Since $B(q)$ is assumed to be full rank, there exists a matrix $A$ of dimension $n-m \times n$, also full rank, such that $A(q) B(q)=0$. Moreover, $\operatorname{span}[A(q)]=\operatorname{ker}(B)=$ $\operatorname{ker}(M)$.

If $\operatorname{dim}(\operatorname{ker} M)=n-2$ then $^{1} f$ can be implemented using a generalized cross product:

$$
f(q)=\sum_{i, j, k \ldots l=0}^{n} \epsilon_{i j k \ldots l}(\nabla \varphi(q))_{j}\left(A(q)_{1}\right)_{k} \cdots\left(A(q)_{n-2}\right)_{l} \hat{e}_{i}
$$

$\epsilon_{i j \ldots l}$ denotes the permutation tensor [5], $\hat{e}_{i}$ are the canonical basis vectors, $A(q)_{i}$ is the $i$ th line of $A(q)$ and $\left(A(q)_{i}\right)_{j}$ is the $j$ th element of line $i$ of $A$. This applies to a fairly extensive class of systems including the unicycle, carts (with or without multiple trailers [11]), etc. In particular, for the RHex motivated planar unicycle described in the examples section, we have $n=3$ and $m=2$. Therefore the vector field $f$ reduces to:

$$
f(q)=\nabla \varphi(q) \times A(q)
$$

Given such a construction for $f$ whose flow moves along level sets, $\Phi_{\alpha}$ of $\varphi$ within the span of $M$, we now seek to reach the stable manifold at the goal of equation (5). Consider the system:

$$
\dot{q}=\sigma(q) f(q)
$$

Where $\sigma: \mathcal{D} \rightarrow \mathbb{R}$ is a scalar function. Any vector field of the form $\sigma(q) f(q)$ verifies the requirements of (7) since $T L^{\prime} \cap \Im(M)$ is a linear space. Suppose we can find a $C^{1}$ scalar function $\mu: \mathcal{D} \rightarrow \mathbb{R}$ such that $\mu^{-1}[0]=\mathcal{W}^{s}$ and $\mu(q)>0, \forall q \notin \mathcal{W}^{s}$. Let $\sigma(q):=-\nabla \mu(q) \cdot f(q)$. If $\nabla \mu(q) \cdot f(q) \neq 0, \forall q \notin \mathcal{W}^{s}$ then the vector field $\sigma(q) f(q)$ is guaranteed take its limit set in $\mathcal{W}^{s}$, as we now show by noting that $\mu$ plays the role of a Lyapunov function for (10):

$$
\begin{aligned}
& \mu>0, \forall q \notin \mathcal{W}^{s} . \\
& \dot{\mu}=\nabla \mu(q) \cdot \dot{q}=-(\nabla \mu(q) \cdot f(q))^{2}<0, \forall q \notin \mathcal{W}^{s}
\end{aligned}
$$

La Salle's theorem states that every solution of (10) approaches the largest invariant subset of $\mathcal{W}^{s}$ as $t \rightarrow \infty$.

${ }^{1}$ If $\operatorname{dim}(\operatorname{ker} M)<n-2$ then one has more directions to move on the level sets of $\varphi$ within the span of $M$, and questions of involutivity arise that lie beyond the scope of the present study. 


\section{Two step controller}

Using the previous notation and constructions, define two vector fields:

$$
\begin{array}{lll}
f_{1}: \mathcal{D} \rightarrow T \mathcal{D} & \text { such that } & f_{1}(q)=-M(q) \cdot \nabla \varphi(q) \\
f_{2}: \mathcal{D} \rightarrow T \mathcal{D} & \text { such that } & f_{2}(q)=\sigma(q) f(q)
\end{array}
$$

Let $f_{1}^{\tau}\left(q_{0}\right)$ and $f_{2}^{\tau}\left(q_{0}\right)$ be the flows generated by $f_{1}$ and $f_{2}$, respectively, i.e., $f^{\tau}\left(q_{0}\right)$ are trajectories of the solution of the differential equation $\dot{q}=f(q)$ with initial condition $q_{0}$, $\tau$ denotes time. Since $\mathcal{D}$ is positive invariant under both $f_{1}$ and $f_{2}$, both of these vector fields take their forward limit sets in $\mathcal{D}$, hence we get two maps $f_{i}^{\infty}: \mathcal{D} \rightarrow \mathcal{D}$ such that:

$$
f_{i}^{\infty}\left(q_{0}\right)=\lim _{\tau \rightarrow \infty} f_{i}^{\tau}\left(q_{0}\right)
$$

Assume that $\mu^{-1}[0]=\mathcal{W}^{s}$. Knowing that for all $q_{0} \notin$ $\mathcal{W}^{c}$ we have $\left\|f_{2}\left(q_{0}\right)\right\|>0$, then $f_{2}^{\infty}\left(q_{0}\right) \in \mathcal{W}^{s}$. But $f_{1}^{\infty}\left(\mathcal{W}^{s}\right)=q^{*} \Rightarrow f_{1}^{\infty} \circ f_{2}^{\infty}\left(q_{0}\right)=q^{*}$. Therefore, for any initial condition outside the center manifold $\mathcal{W}^{c}$ of system (5), applying controller $f_{2}$ followed by controller $f_{1}$ reaches any neighborhood of the goal $q^{*}$, so long $\tau$ is made large enough.

\section{Iterative controller when $\mathcal{W}^{s}$ is unknown}

In general it may not be easy to find a closed form representation of the stable manifold $\mathcal{W}^{s}$. A sufficient but not necessary condition for convergence to the goal is that we can find an approximation of the stable manifold of the form $\widehat{\mathcal{W}}^{s}=\left\{q \in \mathbb{R}^{n}: \hat{\mu}(q)=0\right\}$, such that $\left.\hat{\varphi}=\left.\varphi\right|_{\mathcal{N}\{\widehat{\mathcal{W}}}\right\}$ (the restriction of $\varphi$ to an open neighborhood of $\widehat{W}^{s}$ ) is a Lyapunov function.

Proposition 1: For every initial condition outside the center manifold $\mathcal{W}^{c}$ of system (5), applying $f_{1}$ followed by $\hat{f}_{2}$ intermittently converges to the goal $q^{*}$.

Proof: Let $f_{2}^{\tau}$ be the flow generated by $\hat{\sigma}(q) f(q)$. Let $P_{\tau_{1}, \tau_{2}}(q)=\hat{f}_{2}^{\tau_{2}} \circ f_{1}^{\tau_{1}}(q) \in \mathcal{N}\left\{\widehat{\mathcal{W}}^{s}\right\}$ by choosing a sufficiently large $\tau_{2}$. If $\tau_{i}$ is made a function of $q$, i.e., $\tau_{i}: \mathcal{D} \rightarrow \mathbb{R}^{+}$then define the recursive time-invariant equation:

$q_{k+1}=P_{\tau_{1}, \tau_{z}}\left(q_{k}\right)=\hat{f}_{2}^{\tau_{2}\left(q_{k}\right)} \circ f_{1}^{\tau_{1}\left(q_{k}\right)}\left(q_{k}\right)=P\left(q_{k}\right)$. We are now ready to apply the standard Lyapunov analysis for autonomous discrete-time systems.

Claim 1: $\forall_{0<r_{1}\left\{q_{k}\right)<\infty}, \quad \forall q_{k} \in \mathcal{N}\left\{\widehat{\mathcal{W}}^{s}\right\} / \mathcal{W}^{c}$ then $\hat{\varphi}\left(q_{k+1}\right)<\hat{\varphi}\left(q_{k}\right)$

Proof: Since $q_{k} \notin \mathcal{W}^{c}$ then $\left\|M \cdot \nabla \varphi \circ\left(q_{k}\right)\right\|>0$. Equation (6) guarantees that $f_{1}^{\tau_{1}\left(q_{k}\right)}\left(q_{k}\right) \neq q_{k}, \forall \tau_{\mathbf{l}}\left(q_{k}\right)>$ 0 and $\varphi\left(f_{1}^{\tau_{1}\left(g_{k}\right)}\left(q_{k}\right)\right)<\varphi\left(q_{k}\right)$. Then we get:

$$
\begin{aligned}
\hat{\varphi}\left(q_{k+1}\right) & =\varphi\left(\hat{f}_{2}^{\tau_{2}\left(q_{k}\right)} \circ f_{1}^{\tau_{1}\left(q_{k}\right)}\left(q_{k}\right)\right) \\
& =\varphi\left(f_{1}^{\tau_{1}\left(q_{k}\right)}\left(q_{k}\right)\right)<\varphi\left(q_{k}\right)=\hat{\varphi}\left(q_{k}\right)
\end{aligned}
$$

For a scalar valued function, $v: \mathcal{D} \rightarrow \mathbb{R}$, and a map, $P^{\prime}: \mathcal{D} \rightarrow \mathcal{D}$, define the "discrete derivative" $\Delta v:=v \circ P^{\prime}-v$. The Lyapunov criteria for discrete-time systems states that the origin of $p_{k+1}=P^{\prime}\left(p_{k}\right)$ is asymptotically stable if, in a neighborhood of the origin, there is a continuous positive definite function $v(p)$ so that $\Delta v(p)$ is negative definite. Make $v(p)=\hat{\varphi}(p), P^{\prime}\left(p_{k}\right)=P\left(p_{k}+q^{*}\right)$ and $p=q-q^{*}$.

\section{APPROXIMATING STABLE MANIFOLDS}

In this section we find $k$-order local approximations to the stable manifold at the goal of system (5), by recursively solving a parameter matching equation. In particular, the curvature of the stable manifold at the equilibrium point can be obtained as a function of partial derivatives of the vector field $h(q)$ described next [6]. We start by "normalizing" the system so that the goal is at the origin and the tangent of the stable manifold is spanned by vectors of the canonical base. In doing this, we seek to represent the stable manifold explicitly as $\left[\begin{array}{lll}x_{m+1} & \cdots & x_{n}\end{array}\right]^{T}=$ $g\left(x_{1}, \ldots, x_{m}\right)$. Consider the system:

$$
\dot{q}=-M(q) \nabla \varphi(q)=h(q)
$$

Apply a change of coordinates to get:

$$
\dot{p}=f(p)=R^{-1} h\left(R^{-1} p-q^{*}\right)
$$

Let $J=D f(0)$. The Center Manifold Theorem for Flows states that the eigenspace generated by the eigenvectors with negative eigenvalues of $J$ is tangent to the stable manifold $\mathcal{W}^{s}$ at the origin. Let $R^{\prime}$ be the change of basis for the real Jordan canonical decomposition:

$$
J=R^{\prime-1}\left[\begin{array}{cccc}
\Delta_{1} & 0 & \cdots & 0 \\
0 & \ddots & & \\
\vdots & & \Delta_{p} & \\
0 & & & 0
\end{array}\right] R^{\prime}=R^{\prime-1} \Delta R^{\prime}
$$

where $\Delta_{i}$ are real eigenvalue blocks. Notice that $\Delta_{i}$ are sorted so that the zero eigenvalues are on the bottom. Next, apply the Gram Schmidt ortogonalization to find a rotation matrix $R=\operatorname{Gram}\left(R^{\prime}\right)$. At this point the tangent to the stable manifold of system (12) $\left(\mathcal{W}^{s}\right)$ is the span of the canonical base vectors $\left[\hat{e}_{1}, \ldots, \hat{e}_{m}\right]$. Let $p=\left(x_{1}, \ldots, x_{n}\right)$. Define the function $G: \mathbb{R}^{n} \rightarrow \mathbb{R}^{n-m}$ such that $G(p)=$ $g\left(x_{1}, \ldots, x_{m}\right)-\left[x_{m+1} \cdots x_{n}\right]^{T}$, with $g: \mathbb{R}^{m} \rightarrow \mathbb{R}^{n-m}$. Let $G=0$ be the implicit representation of $\mathcal{W}^{s}$ at the origin and $\left[\begin{array}{lll}x_{m+1} & \cdots & x_{n}\end{array}\right]^{T}=g\left(x_{1}, \ldots, x_{m}\right)$ its explicit representation. We proceed by finding a polynomial approximation of $g$ on partial derivatives of $f$. Let $\hat{g}_{k}$ and $f_{k}$ be $k$-order taylor approximations of $g$ and $f$ at the origin:

$$
\begin{aligned}
& \hat{g}_{k}\left(x_{1}, \ldots, x_{m}\right)=\sum_{\substack{i_{1}, \ldots, i_{m} \geq 0 \\
i_{1}+\cdots+i_{m} \leq k}} \frac{x_{1}^{i_{1}} \cdots x_{m}^{i_{m}}}{i_{1} ! \cdots i_{m} !} \gamma_{i_{1}, \ldots, i_{m}} \\
& \hat{f}_{k}\left(x_{1}, \ldots, x_{n}\right)=\left.\sum_{\substack{i_{1} \\
i_{1}, \ldots, i_{n} \geq 0 \\
i_{1}+\cdots+i_{n} \leq k}} \frac{x_{1}^{i_{1}} \cdots x_{n}^{i_{n}}}{i_{1} ! \cdots i_{n} !}\left(\frac{\partial^{i_{1}}}{\partial x_{1}^{i_{1}}} \cdots \frac{\partial^{i_{n}}}{\partial x_{n}^{i_{n}}} f\right)\right|_{0}
\end{aligned}
$$

Since $G=0$ is an invariant manifold of (12), its total derivative is zero:

$$
\dot{G}=[D g-I] \cdot f=0
$$




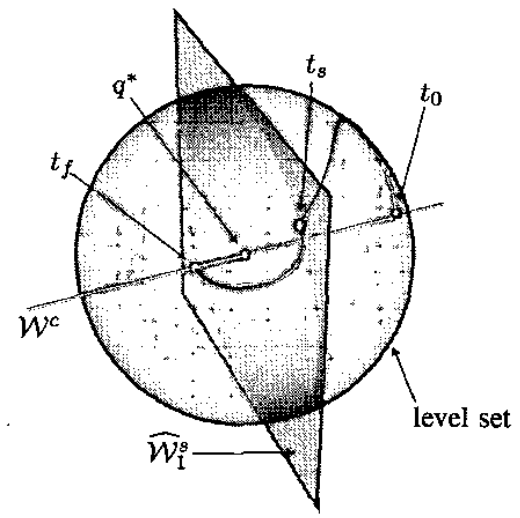

(a) 1st order approximation of stable manifold

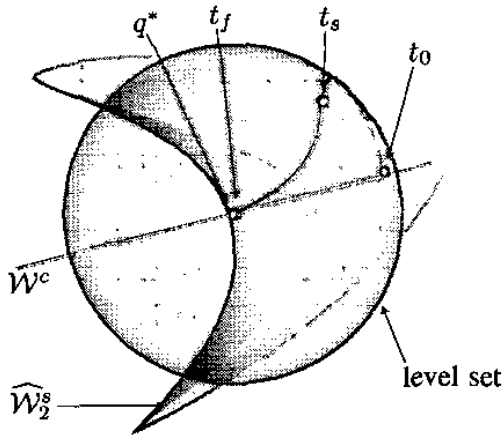

(b) 2nd order approximation of stable manifold

Fig. 1. Simulation of a two step controller using different order approximations of the stable manifold. The initial position is $t_{0}$. The controller switches at $t_{s}$ and the final position is $t_{f}$. Due to the high curvature of the stable manifold $\mathcal{W}^{s}$ at the origin the 1st order approximation requires a higher number of iterations to reach the proximity of the goal.

To find an $k$-order approximation of $\mathcal{W}^{s}$ replace $g$ and $f$ by $\hat{g}_{k}$ and $\hat{f}_{k}$ on equation (13):

$$
\left([D g-I] \cdot \hat{f}_{k}\right) \circ\left(x_{1}, \ldots, x_{m}, \hat{g}_{k}\left(x_{1}, \ldots, x_{m}\right)\right)=0
$$

(14) is a system of $n-m$ equations with variables $x_{1}, \ldots, x_{m}$. By construction, the 0 and 1 st order coefficients are equal to zero. The 2 nd and upper order coefficients are computed recursively by matching the $x_{i}$ coefficients of equation (14), in effect, solving linear equations recursively.

\section{A. Example 1: $\mathcal{W}^{s}$ is a surface on $\mathbb{R}^{3}$}

Here we have $n=3$ and $m=2$, therefore (14) reduces to one equation:

$\Gamma=\left(\left(\hat{f}_{k}\right)_{1} \frac{\partial \hat{g}_{k}}{\partial x}+\left(\hat{f}_{k}\right)_{2} \frac{\partial \hat{g}_{k}}{\partial y}-\left(\hat{f}_{k}\right)_{3}\right) \circ\left(x, y, \hat{g}_{k}(x, y)\right)$

The 2nd order coefficients can be computed by solving the linear set of equations:

$$
\left.\left(\frac{\partial^{2} \Gamma}{\partial x^{2}}=0 \wedge \frac{\partial^{2} \Gamma}{\partial x \partial y}=0 \wedge \frac{\partial^{2} \Gamma}{\partial y^{2}}=0\right)\right|_{x, y=0}
$$

Resulting in:

$$
\left[\begin{array}{c}
\gamma_{0,2} \\
\gamma_{1,1} \\
\gamma_{2,0}
\end{array}\right]=\left.\left[\begin{array}{ccc}
\frac{\partial f_{2}}{\partial y} & \frac{\partial f_{1}}{\partial y} & 0 \\
\frac{\partial f_{2}}{\partial x} & \frac{\partial f_{1}}{\partial x}+\frac{\partial f_{2}}{\partial y} & \frac{\partial f_{1}}{\partial y} \\
0 & \frac{\partial f_{2}}{\partial x} & \frac{\partial f_{2}}{\partial x}
\end{array}\right]^{-1}\left[\begin{array}{c}
\frac{1}{2} \frac{\partial^{2} f_{3}}{\partial y^{2}} \\
\frac{\partial^{2} f_{3}}{\partial x \partial y} \\
\frac{1}{2} \frac{\partial^{2} f_{3}}{\partial x^{2}}
\end{array}\right]\right|_{\substack{x=0 \\
y=0}}
$$

The 3rd and higher order coefficients are computed in a similar fashion. Note that the curvature of $\mathcal{W}^{s}$ at the origin is computed by $\gamma_{1,1}^{2}-\gamma_{0,2}, \gamma_{2,0}$.
B. Example 2: approximations of $\mathcal{W}^{s}$ with high curvature for a unicycle

If $\mathcal{W}^{s}$ has a high curvature at the goal, then the order of approximation becomes relevant, especially if the number of steps necessary to reach a fixed neighborhood of the goal is desired to be minimized. Let $\varphi: \mathbb{R}^{3} \rightarrow \mathbb{R}^{3}$ be a potential function with goal at the origin and $M$ be the nonholonomic projection matrix for the unicycle:

$$
\begin{aligned}
\varphi(x, y, \theta) & =x^{2}+y^{2}+\theta^{2} \\
M(x, y, \theta) & =B \cdot B^{\dagger}=\left[\begin{array}{ccc}
\sin ^{2} \theta & \cos \theta \sin \theta & 0 \\
-\cos \theta \sin \theta & \cos ^{2} \theta & 0 \\
0 & 0 & 1
\end{array}\right]
\end{aligned}
$$

The $\operatorname{ker} M$ is the span of $A(q)=[\cos (\theta) \sin (\theta) 0]$. Using the potential function (15) with $M$ results in the the center manifold $\mathcal{W}^{c}=\left\{(x, y, \theta) \in \mathbb{R}^{3} \mid y=0 \wedge \theta=0\right\}$. Using equation (14) we get the following $k$-th order approximations to the stable manifold at the origin:

$$
\begin{aligned}
\widehat{\mathcal{W}}_{1}^{s} & =\{x=0\} \\
\widehat{\mathcal{W}}_{2}^{s} & =\left\{x=-\frac{y \theta}{2}\right\} \\
& \ldots \\
\widehat{\mathcal{W}}_{6}^{s} & =\left\{x=-\frac{y \theta}{2}-\frac{y \theta^{3}}{48}-\frac{y \theta^{5}}{480}\right\}
\end{aligned}
$$

Figure 1 illustrates numerical simulations of the vector fields (11) using 1st and 2nd order approximations of $\mathcal{W}^{s}$. Due to the high curvature of $\mathcal{W}^{s}$ at the origin, the 1st order approximation results in a poor final position after one iteration. More iterations are required to reach the goal. The 2nd order controller reaches the proximity of the goal in one iteration.

\section{Simulations}

This section provides numerical simulations of two instances of navigation with visual constraints. 

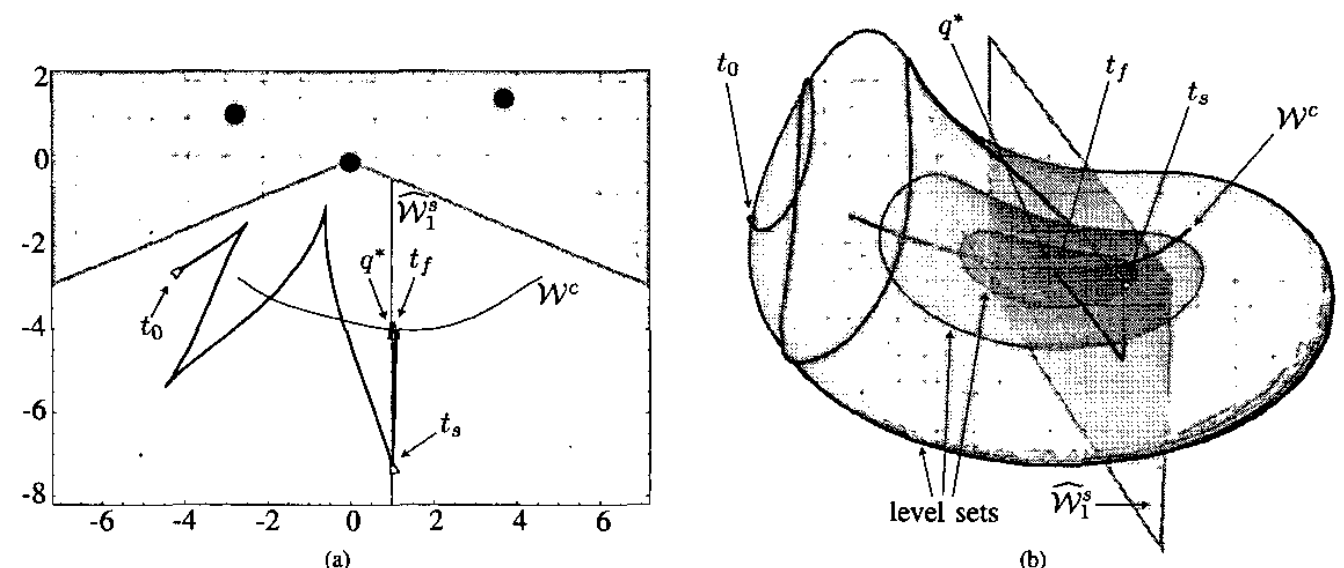

Fig. 2. Simulation of a unicycle using a navigation function introduced by Cowan. The initial position is $t_{0}$. The controller switches at $t_{s}$ and the final position is $t_{f}$. A 1st order approximation of the stable manifold $\mathcal{W}^{s}$ is used. Due to the low curvature of $\mathcal{W}^{s}$ the robot reaches the proximity of the goal in two steps. a) Top view. The beacons are represented by the large black dots. The gray areas violate the visual constraint obstacles. b) Configuration space plotted on $(x, y, 5 \theta-5 \arctan (y / x))$ for legibility purposes.

\section{A. Example 1: registration of robot using set of 3 beacons.}

Cowan et al. [4] introduced the problem of a robot registering itself against a set of 3 beacons. A smooth change of coordinates $\left(h: \mathbb{R}^{3} \rightarrow \mathbb{R}^{3}\right.$ ) maps the projection of each beacon on the image plane to the robot's location in $S E(3)$ (for detailed information on $h$ see [12]). A navigation function $\varphi$ is built in the image plane taking into account the two types of vision constraints:

1) Field of view obstacle: the coordinates of the projected beacons are bounded.

2) Self-occlusion: the coordinates of the projected beacons are not allowed to intercept.

Consider the following potential function:

$\tilde{\varphi}:=\frac{\left(\left(\zeta_{1}-\zeta_{1}^{*}\right)^{2}+\left(\zeta_{2}-\zeta_{2}^{*}\right)^{2}+\left(\zeta_{3}-\zeta_{3}^{*}\right)^{2}\right)^{k}}{\left(\zeta_{M}-\zeta_{1}\right)\left(\zeta_{1}-\zeta_{2}\right)\left(\zeta_{2}-\zeta_{3}\right)\left(\zeta_{3}-\zeta_{m}\right)\left(\zeta_{1}-\zeta_{3}+\zeta_{d}\right)}$

Where $\zeta_{M}$ and $\zeta_{m}$ are the Field of View obstacles and $\zeta_{d}$ is introduced to limit the distance away from the set of beacons. $k$ is a "shaping" parameter. $\tilde{\varphi}$ by construction explodes at the obstacles and is zero at the goal. The resulting navigation function is a squashed version of $\tilde{\varphi}$ :

$$
\varphi\left(\zeta_{1}, \zeta_{2}, \zeta_{3}\right):=\frac{\tilde{\varphi}\left(\zeta_{1}, \zeta_{2}, \zeta_{3}\right)}{\epsilon+\tilde{\varphi}\left(\zeta_{1}, \zeta_{2}, \zeta_{3}\right)}
$$

The final system uses the pullback of $h$ to bring the velocities $\nabla \varphi$ back to $S E(3)$ :

$$
\dot{q}=D h^{T}(q) \cdot \nabla \varphi \circ h(q)
$$

The previous navigation function was developed for a fully actuated body and implemented on the robot RHex [19]. However, the strength of empirical experience suggests that RHex's horizontal plane behavior is modeled by a quasistatic unicycle. Figure 2 illustrates a simulation of the system (3) using the following set of controllers, where $M$ and $A$ are defined for the unicycle in (16). $\sigma$ is given by a 1 st order approximation of $\mathcal{W}^{s}$.

$$
\begin{aligned}
& f_{1}=-M(q) \cdot D h^{T}(q) \cdot \nabla \varphi \circ h(q) \\
& f_{2}=\sigma(q)\left(D h^{T}(q) \cdot \nabla \varphi \circ h(q)\right) \times A(q)
\end{aligned}
$$

The numerical simulations show that the navigation function introduced by Cowan can be reused with no modifications. Notice that on the plane the robot executes a parallel parking maneuver. Although it is well known that for the unicycle the parallel parking motion is required to move sideways, the trajectory obtained on the plane is a natural consequence of moving on a level set of the navigation function. The navigation function enforces that the robot does not hit the obstacles, since doing that would require puncturing the level sets away from the goal.

B. Example 2: registration of robot using a single beacon.

Kantor and Rizzi [8] solved the problem of positioning a robot in relation to a single engineered beacon by using the notion of Sequential Composition of Controllers [3]. The final approach to the goal is implemented using Ikeda's Variable Constraint Control. Let $h$ be a change of coordinates from $S E(2)$ to double polar coordinates:

$$
\left[\begin{array}{l}
\eta \\
\mu \\
d
\end{array}\right]=h(x, y, \theta)=\left[\begin{array}{l}
\arctan (y / x) \\
\theta-\arctan (y / x) \\
\sqrt{x^{2}+y^{2}}
\end{array}\right]
$$

Obstacles are introduced on the field of view so that the robot maintains a range of distances to the beacon and keeps facing it:

$$
\mu_{m}<\mu<\mu_{M} ; \quad d_{m}<d<d_{M}
$$

Consider the following potential function:

$$
\begin{array}{r}
\tilde{\varphi}:=\frac{\left(2-\cos \left(\eta-\eta^{*}\right)-\cos \left(\mu-\mu^{*}\right)+\left(d-d^{*}\right)^{2}\right)^{k}}{\left(1-\cos \left(\mu-\mu_{m}\right)\right)\left(1-\cos \left(\mu-\mu_{M}\right)\right)} \\
\cdot \frac{1}{\left(d-d_{m}\right)\left(d-d_{M}\right)}
\end{array}
$$




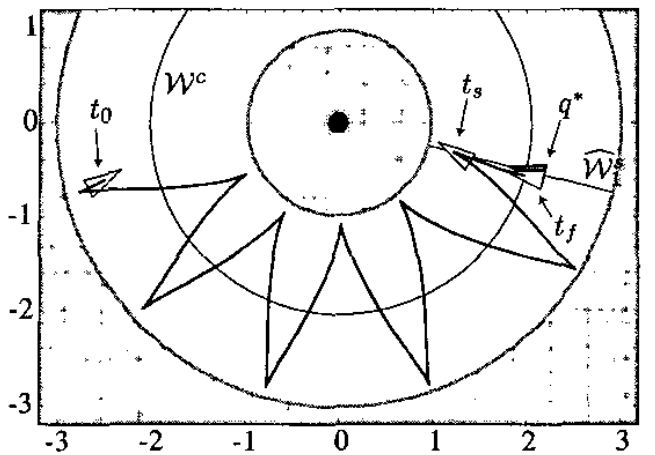

(a)

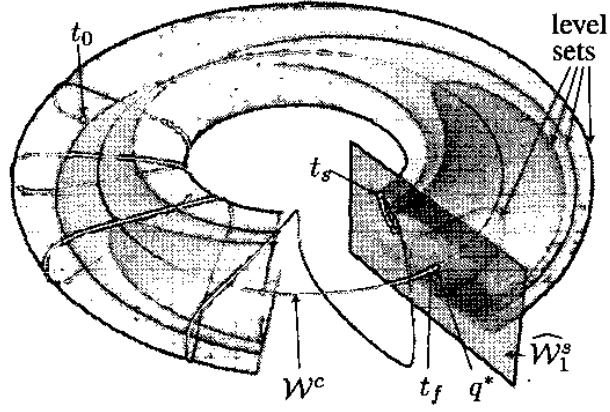

(b)

Fig. 3. Simulation of a unicycle on the visually constrained setup introduced by Kantor and Rizzi. The initial position is $t_{0}$. The controller switches at $t_{s}$ and the final position is $t_{f}$. A 1st order approximation of the stable manifold $\mathcal{W}^{s}$ is used. Due to the low curvature of $\mathcal{W}^{s}$ the robot reaches the proximity of the goal in two steps. a) Top view. The beacon is represented by the large black dot. b) Configuration space plotted in ( $x, y, \mu)$

A squashed version of $\tilde{\varphi}$, as in (17), is used on the controllers (19). Figure 3 illustrates the resulting numerical simulations. Once again, the robot executes the parallel parking maneuver. Simulations suggest that the robot reaches the stable manifold $\mathcal{W}^{s}$ more efficiently if it moves on a level close to the obstacle.

\section{CONCLUSIONS AND FUTURE DIRECTIONS}

This paper introduces the idea of reusing navigation functions developed for fully actuated bodies on motion constrained systems. The resulting switching control law guarantees that the system converges to the goal, even if an approximation of the stable manifold is used. Due to the nature of the switching controller, the obstacles encoded on the navigation function are guaranteed to be avoided. Remaining work includes the implementation of the algorithms presented on the robot RHex on navigation applications.

\section{ACKNOWLEDGMENTS}

We thank George Kantor and Al Rizzi for a number of stimulating and helpful conversations bearing on the problem addressed in this paper. This research was supported by the The University of Michigan, by DARPA/ONR N0001498-1-0747, by DARPA/SPAWAR N660011-03-C-8045 and by Fundação para a Ciência e Tecnologia - Portugal, with the fellowship PRAXIS XXI/BD/18148/98.

\section{REFERENCES}

[1] A. Astolf. Discontinuous control of nonholonomic systems. Systems and Control Letters, 27:37-45, 1996.

[2] R. W. Brockett. Asymptotic stability and feedback stabilization, pages 181-191. Differential Geometric Control Theory. R. W. Brockett, R. S. Millman, and H. J, Sussmann, Birkhauser, Boston, 1983.

[3] R. R. Burridge, A. A. Rizzi, and D. E. Koditschek. Sequential composition of dynamically dexterous robot bchaviors. The International Journal of Robotics Research, 18(6):534-555, June 1999.

[4] Noah J. Cowan, Joel D. Weingarten, and Daniel E. Koditschek Visual servoing via navigation functions. Trans. on Rob. and Aut. 18(4):521-533, August 2002.

[5] H. Goldstein. Classical Mechanics. Addison-Wesley, Reading Mass., 1950.
[6] J. Guckenheimer and P. Holmes. Nonlinear Oscillations, Dynamica Systems, and Bifurcations of Vector Fields. Verlag. New York, 1983.

[7] Takayuki Ikeda, Tsutomu Mita, and Brian Anderson. Variabla constraint control fos a class of nonholonomic systems. Technical report, Department of Mechanical Control and Systems, Tokyo Institute of Technology, 2003.

[8] G. Kantor and A. Rizzi. Scquential composition for control of underactuated systems. Technical Report CMU-RI-TR-03-23, Robotics Institute, Carnegie Mellon, 2003.

[9] $\mathrm{H}$. Khennouf and C. C. de Wit. On the construction of stabilizing discontinuous controllers for nonholonomic systems. In Proc. of IFAC Nonlinear Control Systems Design Sympo., pages 747-752, Tahoe City, USA, 1995.

[10] Daniel E. Koditschek and Elon Rimon. Robot navigation functions on manifolds with boundary. Advances in Applied Mathematics, $11: 412-442,1990$.

[11] Jean-Claude Latombe. Robot Motion Planning. Kluwer Academic Publishers, Boston, 1991.

[12] Gabriel A. D. Lopes and Daniel E. Koditschek. Visual registration and navigation using planar features. In International Conference in Robotics and Automation, Tajpei, Taiwan, 2003. IEEE.

[13] J. Luo and P.Tsiotras. Exponentially convergent control laws for nonbolonomic systems in power form. Systems and Control Letters, $35: 87-95,1998$.

[14] Ezio Malis, Francois Chaumette, and Sylvie Boudet. 2-1/2-d visual servoing. IEEE Transactions on Robotics and Automation, pages 238-250, 1999.

[15] S. Monaco and D. N-Cyrot. An introduction to motion planning using multirate digital control. In Proc. of IEEE Conference on Decision and Control, pages 1780-1785, 1992.

[16] P. Murrieri, D. Fontanelli, and A. Bicchi. Visual-servoed parking with limited view angle, pages 265-274. Expcrimental Robotics VIII, Springer-Verlag, Berlin Heidclberg, 2003.

[17] J-B. Pomet. Explicit design of time-varying stabilizing control laws for a class of controllable systems without drift. Systems and Control Letters, 18:147-158, 1992.

[18] C. Samson. Control of chained systems - application to path following and time-varying pointstabilization of mobile robots. IEEE Trans. Automation and Control, 40(1):64-77, 1995.

[19] U. Saranli, M. Buehler, and D.E. Koditschek. Rhex: A simple and highly mobile hexapod robot. The International Joumal of Robotics Research, 20(7):616-631, 2001.

[20] O. J. Sordalen and O. Egeland. Exponential stabilization of nonholonomic chained systems. IEEE Trans. Automation and Control. 40(1):35-49, 1995

[21] A. Tayebi, M. Tadjine, and A. Rachid. Discontinuous control design for the stabilization of nonholonomic systems in chained form using the backstepping approach. In Proc. of IEEE Conference on Decision and Control, pages 3089-3090, 1997.

[22] Hong Zhang and James P. Ostrowski. Visual servoing with dynamics: Control of an unmanned blimp. In Int. Conf. on Robotics and Automation, 1999. 